\title{
High gaseous nitrous acid (HONO) emissions from light- duty diesel vehicles
}

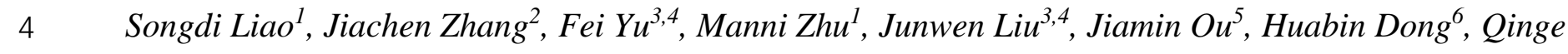

${ }^{2}$ Department of Civil and Environmental Engineering, University of Southern California, Los Angeles,

Corresponding author:

Dr. Junyu Zheng

17 E-mail: zheng.junyu@gmail.com 


\section{Content:}

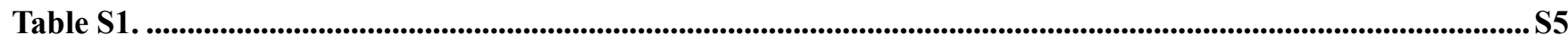

Table S2.

Table S3.

Table S4.

Table S5.

Figure S1.

Figure S2..

.. 58

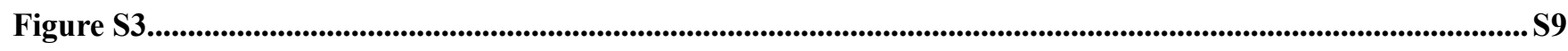

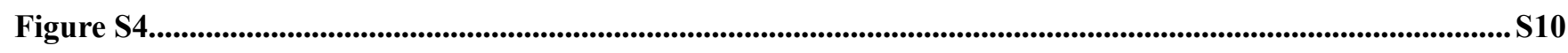

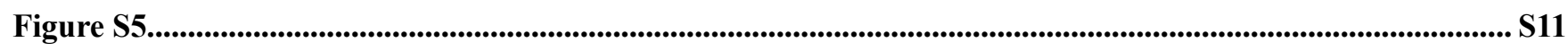

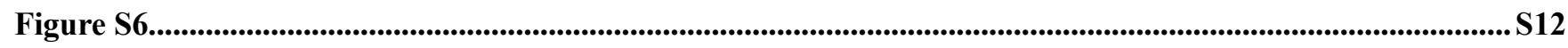

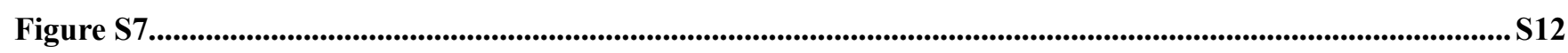

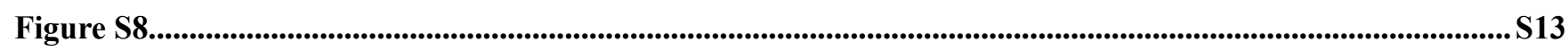

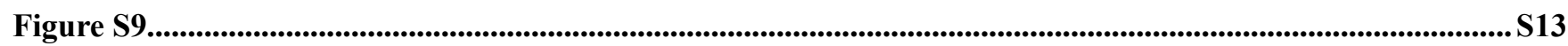



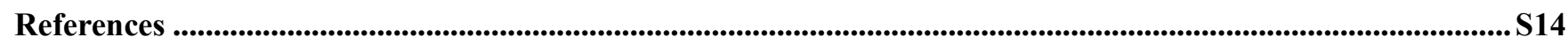


PSDS : In our experiments, the first dilution chamber of PSDS is set at the EFM sampling port with a dilution

ratio of 10 to reduce vehicle exhaust gas temperature to below $60^{\circ} \mathrm{C}$. Then, the second dilution chamber whose

dilution ratio is also set to 10 further reduces the sample gas temperature to ambient air temperature. Finally, the third dilution chamber is used to further reduce HONO concentrations to be lower than the maximum concentrations that can be measured by the HONO detector. After going through first dilution chamber, second dilution chamber, and third dilution chamber, the exhaust gas can be diluted by hundreds to thousands of times. In addition, the dilution ratio of the three chambers can also be easily adjusted for different dilution ratios, and therefore PSDS can be used to measure a wide range of HONO concentrations.

The temperature of the mixed gas after was calculated according to Equation (1)

$$
V_{1} \times\left(T_{1}-\mathrm{T}\right)=V_{2} \times\left(\mathrm{T}-T_{2}\right)
$$

Where $V_{1}$ denotes the sampling flow rate $(0.5 \mathrm{~L} / \mathrm{min}), T_{1}$ denotes the temperature of tailpipe gas (assumed as $250^{\circ} \mathrm{C}$ ), $V_{2}$ denotes the flow rate of nitrogen, $T_{2}$ denotes the temperature of nitrogen (assumed as $20^{\circ} \mathrm{C}$ ), and $\mathrm{T}$ denotes the temperature of mixed gas. The effect of gas density changes is ignored in the calculation. The mixed gas temperature was $47.5^{\circ} \mathrm{C}$ under 10 times dilution ratio (the $\mathrm{MFC} 1$ flow rate of nitrogen was $4.5 \mathrm{~L} / \mathrm{min}$ ), so we set a constant temperature of $60^{\circ} \mathrm{C}$ for the sampling tube. Due to the high flow rate in the sampling tube, the residence time is less than 10s. In addition, we innovatively add a data acquisition and system control module composing of a 7-inch touch panel and a 32-bit ARM Microcontroller (CortexM3 STM32F103VET6) in the PSDS for user operation and data acquisition.

LOPAP: HONO concentrations were measured by long-path absorption photometric (LOPAP) based on Griess-Saltzman reaction. LOPAP includes three main modules: the sampling module, the reaction module, and the detection module. In the sampling module, $\mathrm{HONO}$ was scrubbed by $60 \mathrm{mmol}$ sulfanilamide (SA) in 1 $\mathrm{mol} / \mathrm{L} \mathrm{HCl}$ in stripping coils, whose temperature is maintained at $20^{\circ} \mathrm{C}$ with cycling water cooled by a cooling 
device to ensure that sampling efficiency would not be affected by the ambient temperature variations. The scrubbed nitrite was then derivatized with $0.8 \mathrm{mmol} / \mathrm{L} \mathrm{N}-(1-72$ naphthyl) ethylenediamine dihydrochloride 74 (NEDA) to form an azo dye in the reaction module. Then the azo dye was put into a detection module, which mainly includes a Liquid Waveguide Capillary Cell (LWCC), a light source, and a photodetector. In order to correct the influence of interfering species, LOPAP adjusts two stripping coils in series in the sampling module to capture $\mathrm{HONO}$ and other interfering species (including $\mathrm{NO}_{2}, \mathrm{NO}, \mathrm{O}_{3}, \mathrm{SO}_{2}$, etc.) in the gas phase. The first coil captures both the HONO and the interference signal while the second coil sampler detects the interference signal, which serves as a dynamic zero point. We corrected the HONO concentration by subtracting the calibrated signal of the second coil from the calibrated signal in the first coil in order to remove interferences of $\mathrm{NO}_{2}$ and other gas species on HONO measurements. LOPAP-PKU and LOPAP-JNU were developed and used in this study to measure HONO emissions from vehicle exhaust. LOPAP-PKU has a response time of approximately 120 seconds, data refresh time of 1 second, and detection limit of approximately 20 pptv.

Calculation of emission factors: The emission factors based on fuel use (EFs) are calculated according to the following Equation:

$$
E F_{i}=\frac{m_{i} \times \mathrm{t}}{M_{\text {fuel }}}
$$

Where $\mathrm{EF}_{\mathrm{i}}(\mathrm{g} / \mathrm{kg})$ denotes fuel-based emission factor of pollutant i. $\mathrm{m}_{\mathrm{i}}$ denotes instantaneous mass emissions of pollutants $\mathrm{i}(\mathrm{g} / \mathrm{min}) . \mathrm{t}(\mathrm{min})$ denotes driving time during the test cycle. $\mathrm{M}_{\text {fuel }}(\mathrm{kg})$ denotes fuel used during the test cycle." 
95 Table S1. Specifications of the light-duty diesel trucks (LDDTs)

\begin{tabular}{|c|c|c|c|c|c|c|}
\hline Number & $\begin{array}{l}\text { Emission } \\
\text { standard }\end{array}$ & $\begin{array}{l}\text { Model } \\
\text { year }\end{array}$ & Odometer (km) & $\begin{array}{c}\text { Engine } \\
\text { displacement (L) }\end{array}$ & $\begin{array}{c}\text { Weight } \\
\text { (metric ton) }\end{array}$ & $\begin{array}{c}\text { Aftertreatment } \\
\text { device }\end{array}$ \\
\hline No.1 & China III & 2012 & 370000 & 2.8 & 2.2 & a) \\
\hline No. 2 & China III & 2013 & 173046 & 2.8 & 2.8 & l \\
\hline No.3 & China IV & 2016 & 157982 & 3.8 & 2.7 & l \\
\hline No.4 & China IV & 2016 & 53072 & 2.8 & 2.6 & ${ }^{{ }^{b}} \mathrm{SCR}$ \\
\hline No.5 & China V & 2018 & 55358 & 2.8 & 2.8 & SCR \\
\hline No.6 & China V & 2018 & 12749 & 2.8 & 2.8 & SCR \\
\hline No.7 & China III & 2012 & 190283 & 2.8 & 2.5 & / \\
\hline No.8 & China III & 2013 & 308164 & 2.8 & 2.2 & l \\
\hline No.9 & China III & 2013 & 185713 & 2.8 & 2.8 & l \\
\hline No.10 & China III & 2012 & 251149 & 2.8 & 2.7 & / \\
\hline No.11 & China IV & 2013 & 166200 & 2.8 & 2.8 & SCR \\
\hline No.12 & China IV & 2014 & 185952 & 2.8 & 4.3 & SCR \\
\hline No.13 & China IV & 2015 & 183922 & 2.8 & 2.7 & SCR \\
\hline No.14 & China IV & 2016 & 158629 & 2.8 & 2.2 & SCR \\
\hline No.15 & China V & 2018 & 67296 & 2.8 & 4.5 & SCR \\
\hline No.16 & China V & 2018 & 32268 & 2.6 & 2.3 & SCR \\
\hline No.17 & China V & 2018 & 45587 & 2.6 & 2.3 & SCR \\
\hline
\end{tabular}

Note: ${ }^{\mathrm{a}}$ Missing information. ${ }^{\mathrm{b}}$ Selective Catalytic Reduction system.

98 Table S2. Specifications of the light-duty gasoline vehicles (LDGVs)

\begin{tabular}{cccccc}
\hline Number & Emission standard & Model year & Odometer $(\mathbf{k m})$ & Engine displacement (L) & Weight (metric ton) \\
\hline No.1 & China III & 2005 & 66980 & 1.8 & 1.8 \\
No.2 & China III & 2007 & 7630 & $\mathrm{~N}$ & $\mathrm{~N}$ \\
No.3 & China III & 2006 & $\mathrm{~N}$ & 1.6 & 1.6 \\
No.4 & China III & 2009 & 156324 & 1.6 & 1.6 \\
No.5 & China III & 2009 & 299923 & 2.0 & 2.0 \\
No.6 & China IV & 2010 & 188824 & 2.0 & 2.0 \\
No.7 & China IV & 2010 & 325349 & 2.0 & 2.0 \\
No.8 & China IV & 2012 & 111247 & 1.2 & 1.3 \\
No.9 & China IV & 2010 & 20809 & 1.5 & 1.78 \\
No.10 & China V & 2016 & 94656 & 2.4 & 2.4 \\
No.11 & China V & 2018 & $\mathrm{~N}$ & 2.0 & 1.9 \\
No.12 & China V & 2017 & 107513 & 1.8 & 1.8 \\
No.13 & China V & 2016 & 83509 & 1.5 & 1.5 \\
No.14 & China VI & 2019 & 10263 & 2.0 & 1.9 \\
No.15 & China VI & 2019 & 350 & 1.2 & 1.8 \\
No.16 & China VI & 2019 & 49 & 1.5 & 1.7 \\
\hline
\end{tabular}


Table S3. Comparison of the ER $\mathrm{HONO}_{\mathrm{NOx}}$ measured in this study with previous studies.

\begin{tabular}{|c|c|c|c|c|c|}
\hline Country & Methods & Vehicle type & Measurements & ERHONO/NOx & Reference \\
\hline & Chassis dynamometer & 8 vehicles & DOAS & $0.1 \%-0.8 \%$ & Pitts et al., $1984^{1}$ \\
\hline USA & Tunnel & $67 \%$ LDGV,33\%van & Denuder & $0.29 \pm 0.05 \%$ & Kirchstetter et al., $1996^{2}$ \\
\hline Germany & Tunnel & Main gasoline car & DOAS & $0.3 \%-0.8 \%$ & Kurtenbach et al., $2001^{3}$ \\
\hline Germany & Vertical profile of HONO & & LOPAP & $0.8 \%$ & Kleffmann et al., $2003^{4}$ \\
\hline USA & Highway junction & 5-10\% HDDV & LOPAP & $1.7 \pm 0.09 \%$ & Rappenglück et al.,20135 \\
\hline China & Tunnel & $33 \%$ LDGV & LOPAP & $0.5 \%-1.6 \%$ & $\mathrm{Xu}$ et al., $2015^{6}$ \\
\hline China & Tunnel & & CAC and LOPAP & $2.1 \%$ & Yang et al.,2014 \\
\hline China & Tunnel & $38 \%$ diesel vehicle & LOPAP & $1.24 \pm 0.35 \%$ & Liang et al. $2017^{8}$ \\
\hline UK & Tunnel & $59 \%$ diesel vehicle & BBCEAS & $0.72-1.01 \%$ & Kramer et al. $2020^{9}$ \\
\hline Japan & Chassis dynamometer & LDGV & CEAS & $0.11-0.68 \%$ & Nakashima and Kajii et al.,2017 \\
\hline Japan & Chassis dynamometer & MDGV & ADAMD & $0-0.95 \%$ & Ha T. Trinh et al.,2017 11 \\
\hline Japan & Chassis dynamometer & MDDV & ADAMD & $0.16-1 \%$ & Ha T. Trinh et al.,2017 ${ }^{11}$ \\
\hline China & Chassis dynamometer & LDGV & LOPAP & $0.03-0.42 \%$ & Yuhan Liu et al.,2017 \\
\hline China & Chassis dynamometer & LDGV-China III & LOPAP & $0.007-0.18 \%$ & This study \\
\hline China & Chassis dynamometer & LDGV-China IV & LOPAP & $0.02-0.21 \%$ & This study \\
\hline China & Chassis dynamometer & LDGV-China V & LOPAP & $0.003-0.22 \%$ & This study \\
\hline China & Chassis dynamometer & LDGV-China VI & LOPAP & $0.01-0.30 \%$ & This study \\
\hline China & Chassis dynamometer & LDDT-China III & LOPAP & $2.1-10.3 \%$ & This study \\
\hline China & Chassis dynamometer & LDDT-China IV & LOPAP & $1.2-3.4 \%$ & This study \\
\hline China & Chassis dynamometer & LDDT-China V & LOPAP & $0.1-1.4 \%$ & This study \\
\hline
\end{tabular}

Note: Gray shades highlight the studies that measure HONO emissions from diesel vehicles. 
Table S4. Chinese emission certification information for gasoline and diesel vehicle.

\begin{tabular}{|c|c|c|c|c|c|c|}
\hline \multirow{2}{*}{ Engine Type } & \multirow{2}{*}{ Stage } & \multirow{2}{*}{ Implementation Date } & \multirow{2}{*}{$\frac{\text { Reference Mass }}{\mathrm{kg}}$} & CO & $\mathrm{HC}+\mathrm{NOx}$ & NOx \\
\hline & & & & \multicolumn{3}{|c|}{$\mathrm{g} / \mathbf{k m}$} \\
\hline \multirow{12}{*}{ Spark Ignition (gasoline) } & \multirow{3}{*}{ China III } & \multirow{3}{*}{2007} & $<1305$ & 2.30 & 0.35 & 0.15 \\
\hline & & & $1305-1760$ & 4.17 & 0.43 & 0.18 \\
\hline & & & $>1760$ & 5.22 & 0.5 & 0.21 \\
\hline & \multirow{3}{*}{ China IV } & \multirow{3}{*}{2012} & $<1305$ & 1.00 & 0.18 & 0.08 \\
\hline & & & $1305-1760$ & 1.81 & 0.23 & 0.10 \\
\hline & & & $>1760$ & 2.27 & 0.27 & 0.11 \\
\hline & \multirow{3}{*}{ China V } & \multirow{3}{*}{2017} & $<1305$ & 1.00 & 0.16 & 0.06 \\
\hline & & & $1305-1760$ & 1.81 & 0.205 & 0.075 \\
\hline & & & $>1760$ & 2.27 & 0.242 & 0.082 \\
\hline & \multirow{3}{*}{ China VI } & \multirow{3}{*}{2019} & $<1305$ & 0.7 & 0.16 & 0.06 \\
\hline & & & $1305-1760$ & 0.88 & 0.205 & 0.075 \\
\hline & & & $>1760$ & 1.00 & 0.242 & 0.082 \\
\hline \multirow{9}{*}{ Compression Ignition (Diesel) } & \multirow{3}{*}{ China III } & \multirow{3}{*}{2007} & $<1305$ & 0.64 & 0.56 & 0.50 \\
\hline & & & $1305-1760$ & 0.80 & 0.72 & 0.65 \\
\hline & & & $>1760$ & 0.95 & 0.86 & 0.78 \\
\hline & \multirow{3}{*}{ China IV } & \multirow{3}{*}{2012} & $<1305$ & 0.50 & 0.30 & 0.25 \\
\hline & & & $1305-1760$ & 0.63 & 0.39 & 0.33 \\
\hline & & & $>1760$ & 0.74 & 0.46 & 0.39 \\
\hline & \multirow{3}{*}{ China V } & \multirow{3}{*}{2017} & $<1305$ & 0.50 & 0.23 & 0.18 \\
\hline & & & $1305-1760$ & 0.63 & 0.295 & 0.235 \\
\hline & & & $>1760$ & 0.74 & 0.35 & 0.28 \\
\hline
\end{tabular}

Table S5. Comparisons of $\mathrm{HONO}, \mathrm{NO}$ and $\mathrm{NO}_{2}$ emission factors of two test cycle.

\begin{tabular}{cccccccc}
\hline \multirow{2}{*}{ Standard } & \multicolumn{3}{c}{ ECE } & & & SSM \\
\cline { 2 - 4 } & HONO $(\mathbf{g} / \mathbf{k g})$ & $\mathbf{N O}(\mathbf{g} / \mathbf{k g})$ & $\mathbf{N O}_{2}(\mathbf{g} / \mathbf{k g})$ & & HONO $(\mathbf{g} / \mathbf{k g})$ & NO $(\mathbf{g} / \mathbf{k g})$ & $\mathbf{N O}(\mathbf{g} / \mathbf{k g})$ \\
\hline China III & $3.22 \pm 1.23$ & $18.56 \pm 1.60$ & $13.13 \pm 0.057$ & & $1.16 \pm 0.48$ & $27.91 \pm 4.70$ & $11.62 \pm 3.14$ \\
China IV & $0.79 \pm 0.27$ & $23.58 \pm 4.11$ & $3.53 \pm 0.16$ & & $0.50 \pm 0.22$ & $24.46 \pm 4.57$ & $7.37 \pm 7.21$ \\
China V & $0.32 \pm 0.13$ & $31.31 \pm 0.57$ & $0.44 \pm 0.042$ & & $0.078 \pm 0.013$ & $32.66 \pm 4.12$ & $0.72 \pm 0.20$ \\
\hline
\end{tabular}




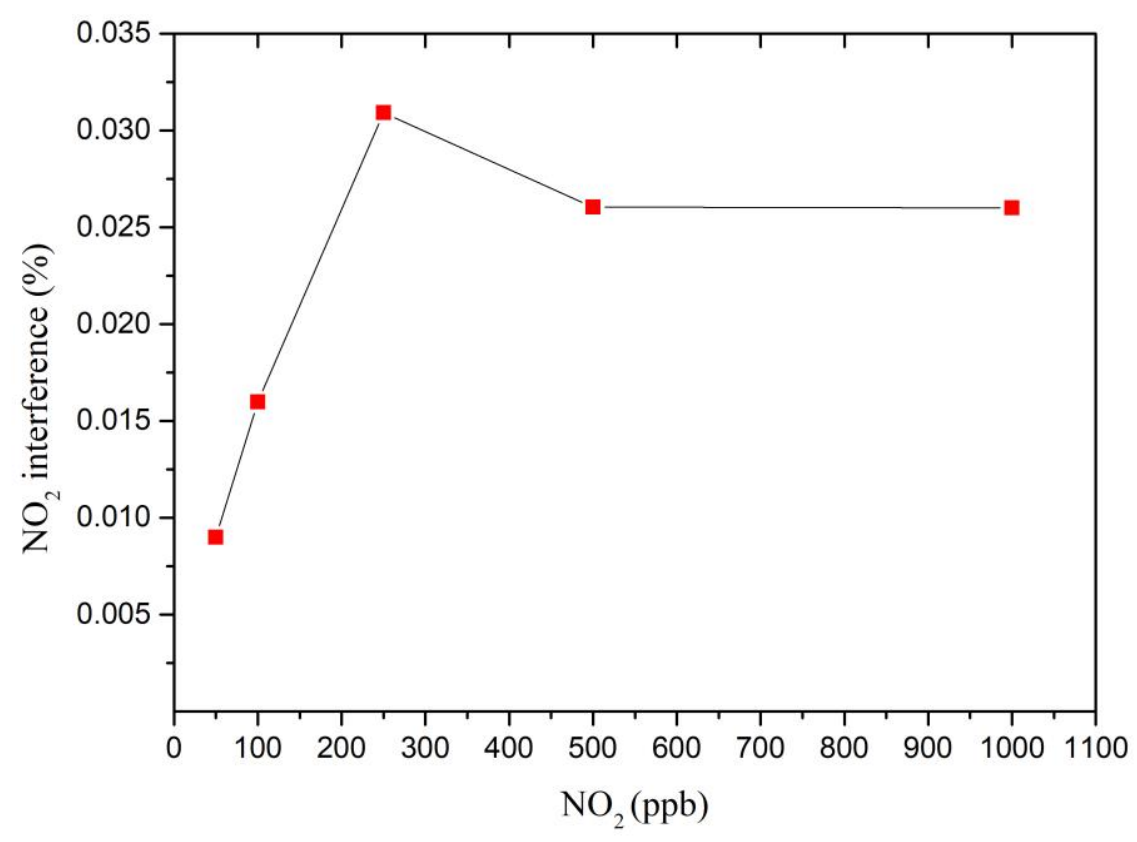

Figure S1. The relationship between $\mathrm{NO}_{2}$ interference of the LOPAP and $\mathrm{NO}_{2}$ concentrations in exhaust

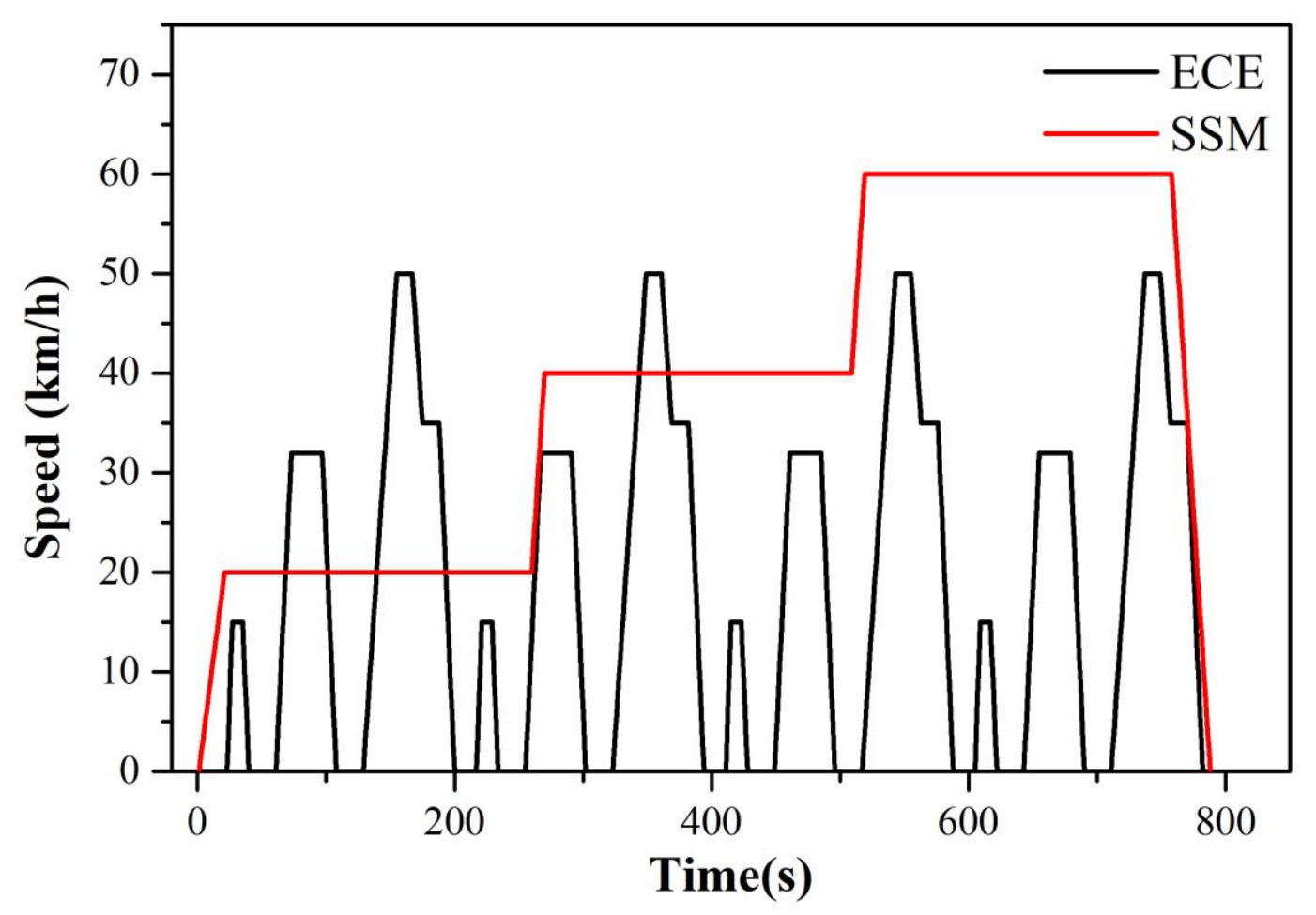

Figure S2. Speed profile of the Economic Commission for Europe (ECE) test cycle and a customized 

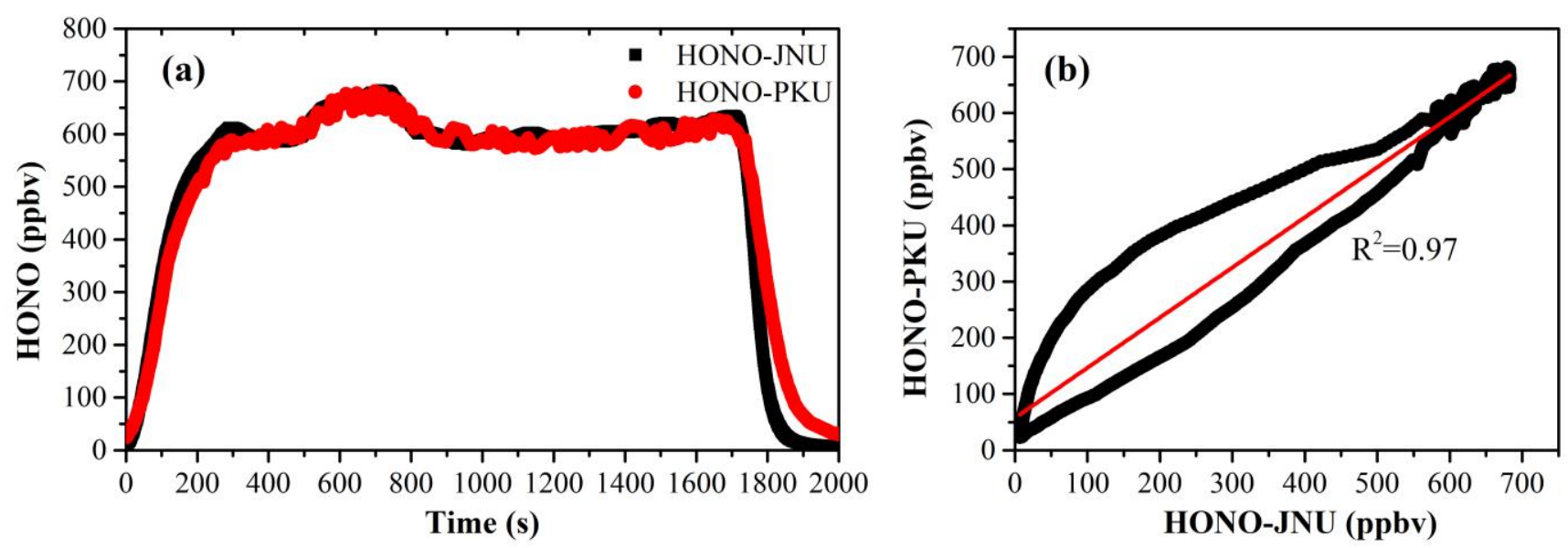

Figure S3. (a) Time series of HONO concentrations and (b) correlation of HONO concentrations measured by two LOPAPs (HONO-JNU and HONO-PKU) for an LDDT. 

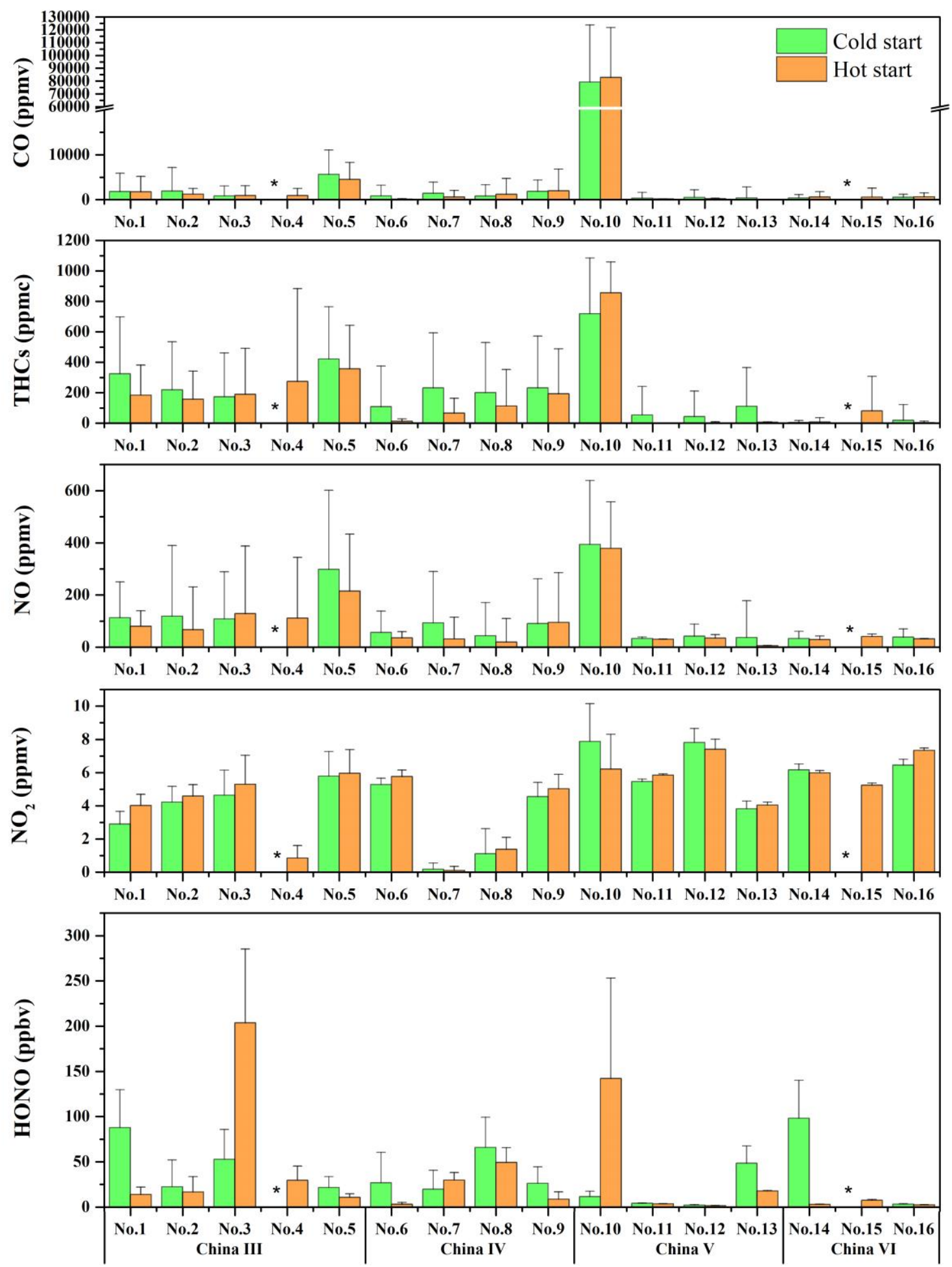

Figure S4. Summary of average $\mathrm{CO}$, THCs, $\mathrm{NO}, \mathrm{NO}_{2}$ and $\mathrm{HONO}$ concentrations from LDGVs for ECE 

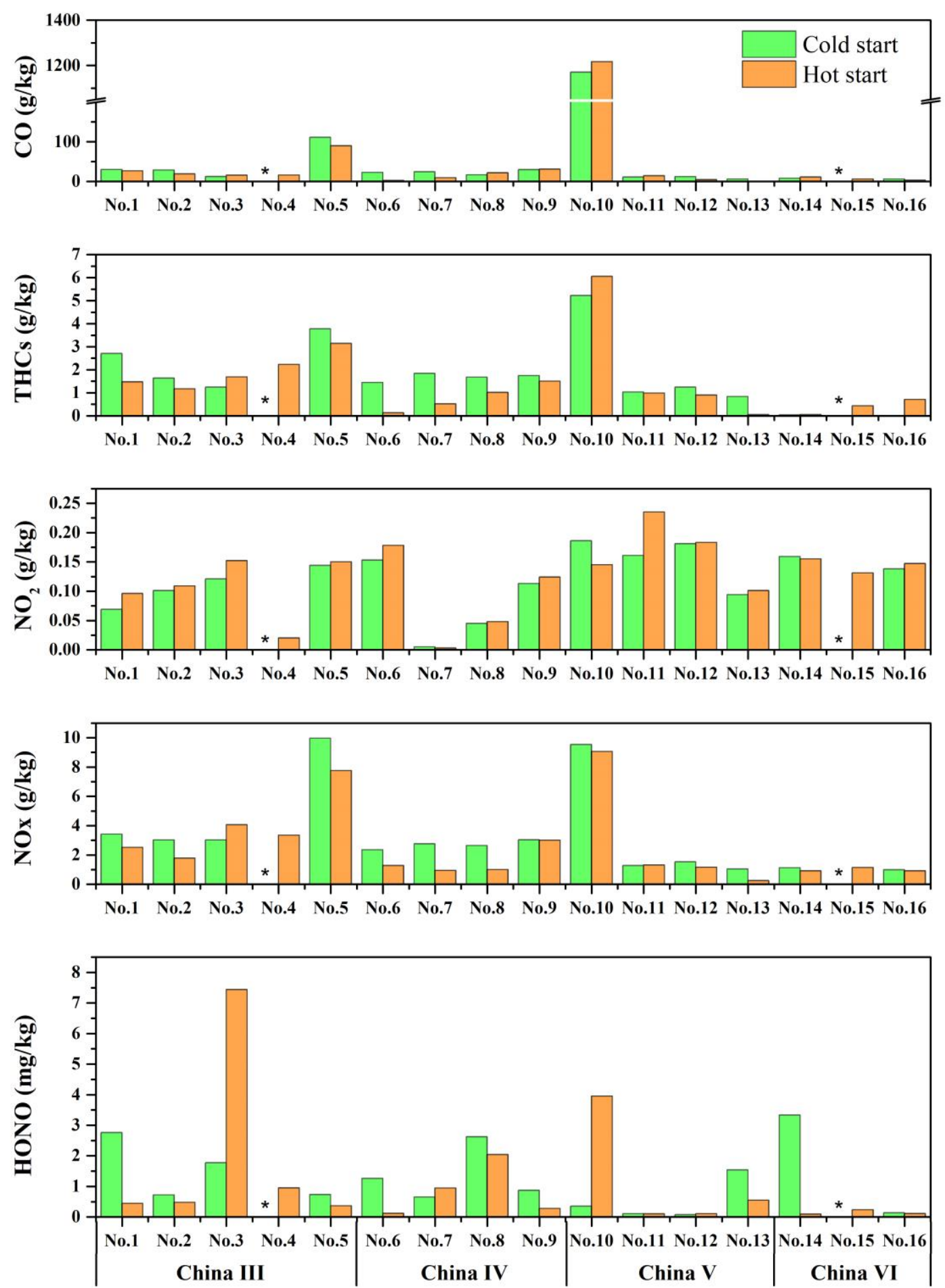

Figure S5. Summary of CO, THCs, NO, $\mathrm{NO}_{\mathrm{X}}$, and HONO EFs from LDGVs for ECE driving cycle. The 

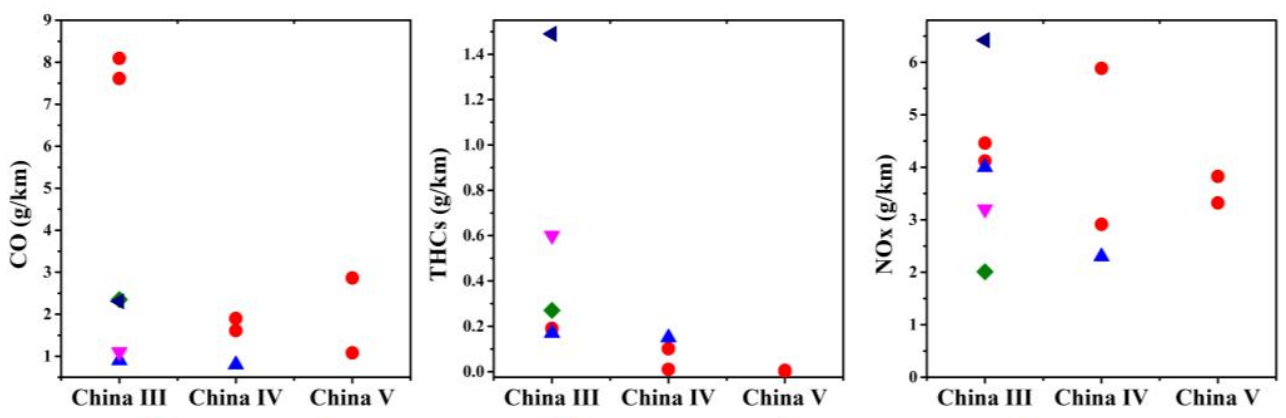

- This study $\Delta$ Yao et al. (2015) $\nabla$ Guo et al. (2014) $\diamond$ Hou et al. (2010) 4 Xue et al. (2010)

Figure S6. Comparison of the CO, THCs and NOx emission factors (based on distance) of LDDT measured in this study with previous studies. ${ }^{13-16}$
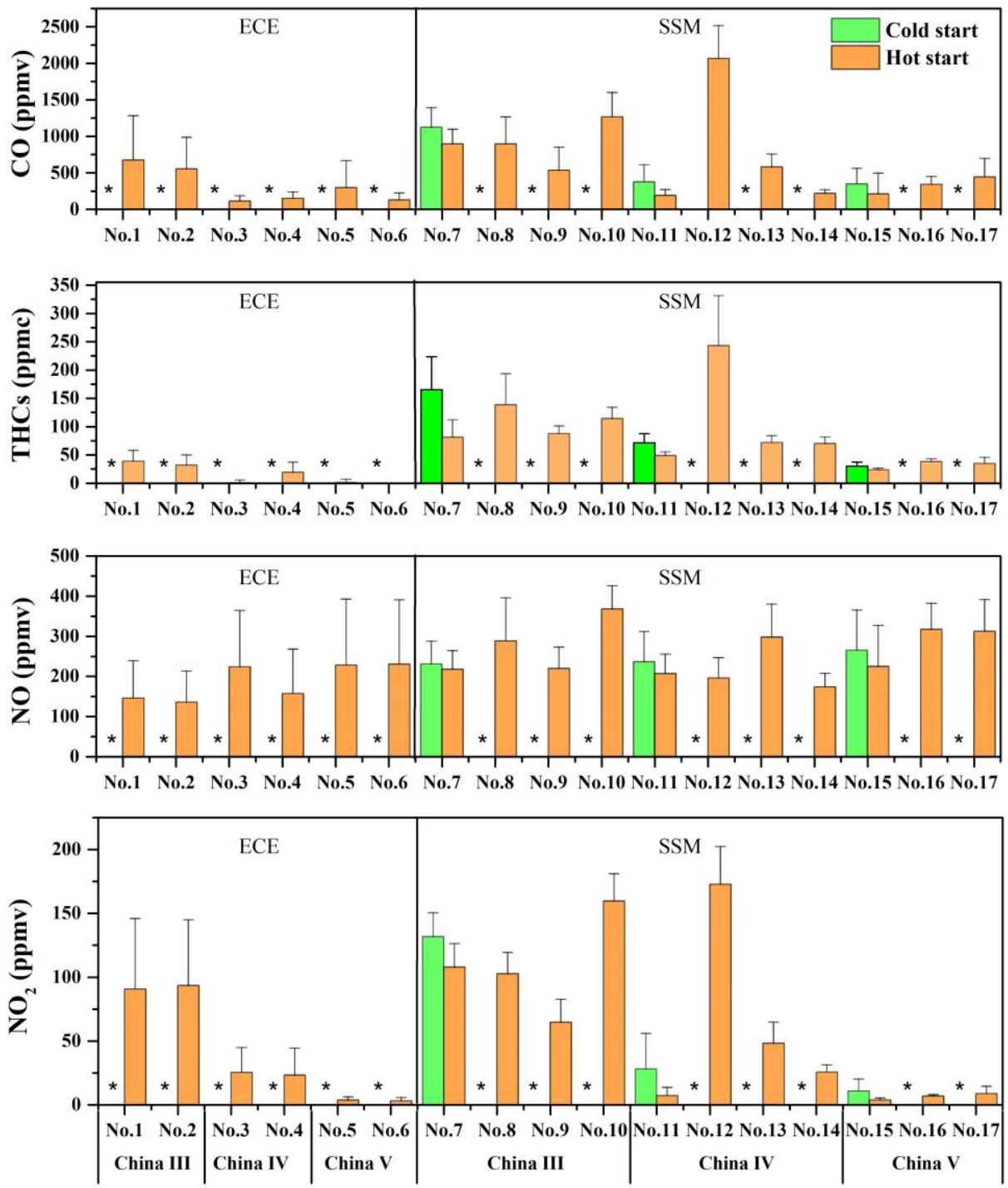

Figure S7. Summary of average $\mathrm{CO}$, THCs, $\mathrm{NO}$, and $\mathrm{NO}_{2}$ concentrations from LDDTs for two driving

cycles. The symbol "**" means "not tested." 

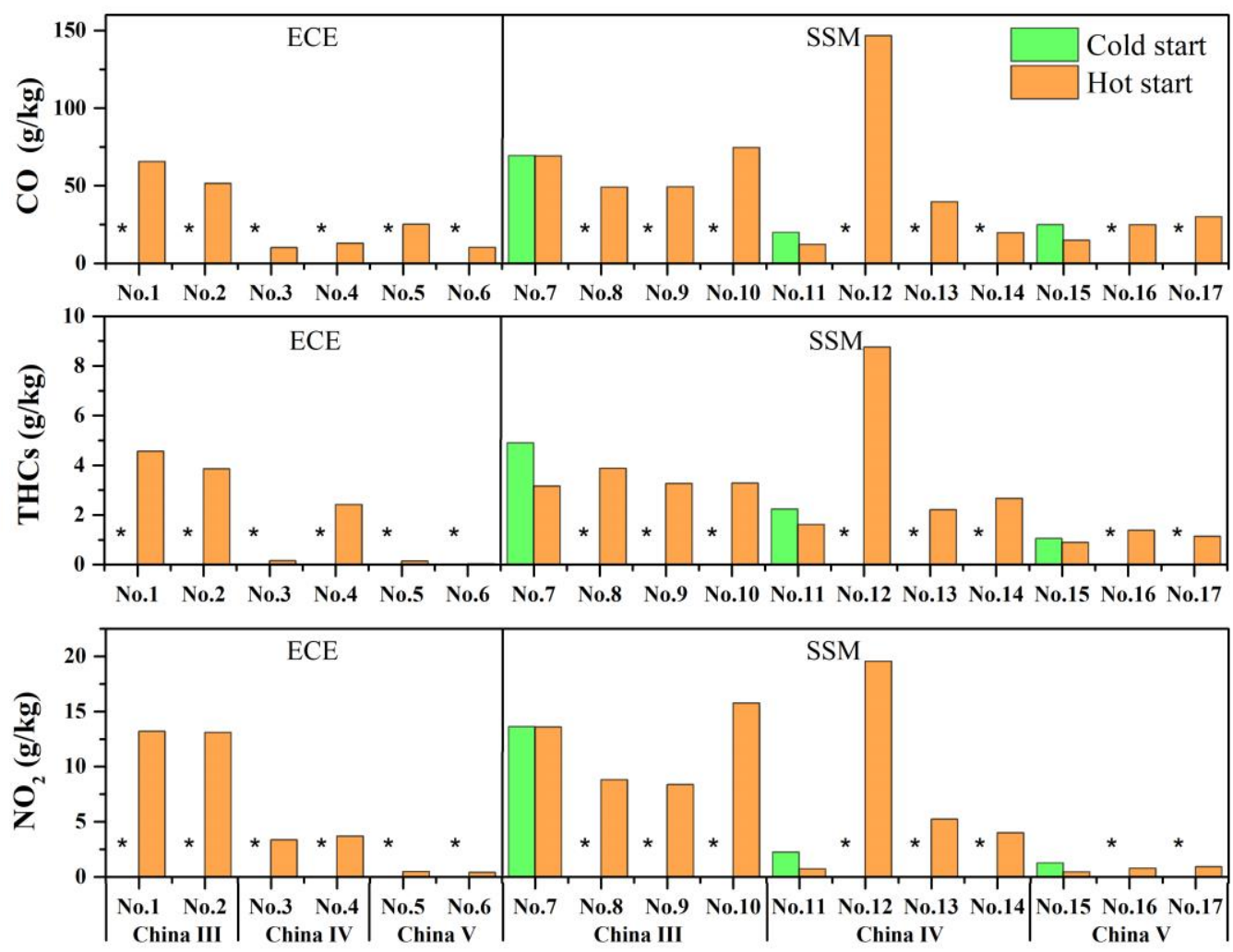

Figure S8. Summary of CO, THCs, and $\mathrm{NO}_{2}$ EFs from LDDTs for two driving cycles. The symbol “*” means "not tested."

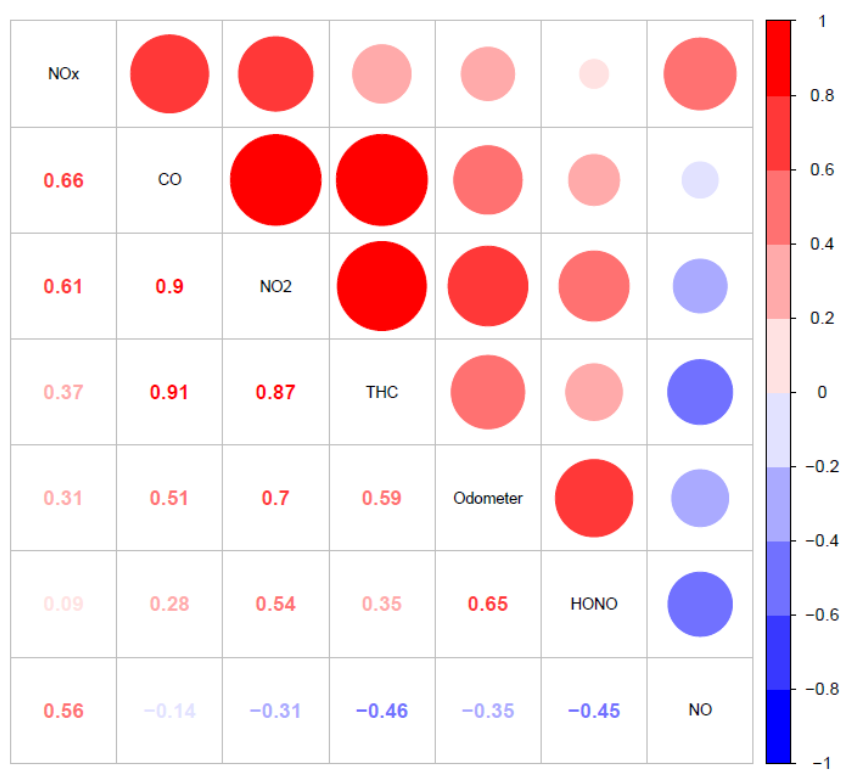

Figure S9. Correlation between HONO EFs and regulated gaseous pollutant EFs (and vehicle odometer) at hot start mode from LDDT. 

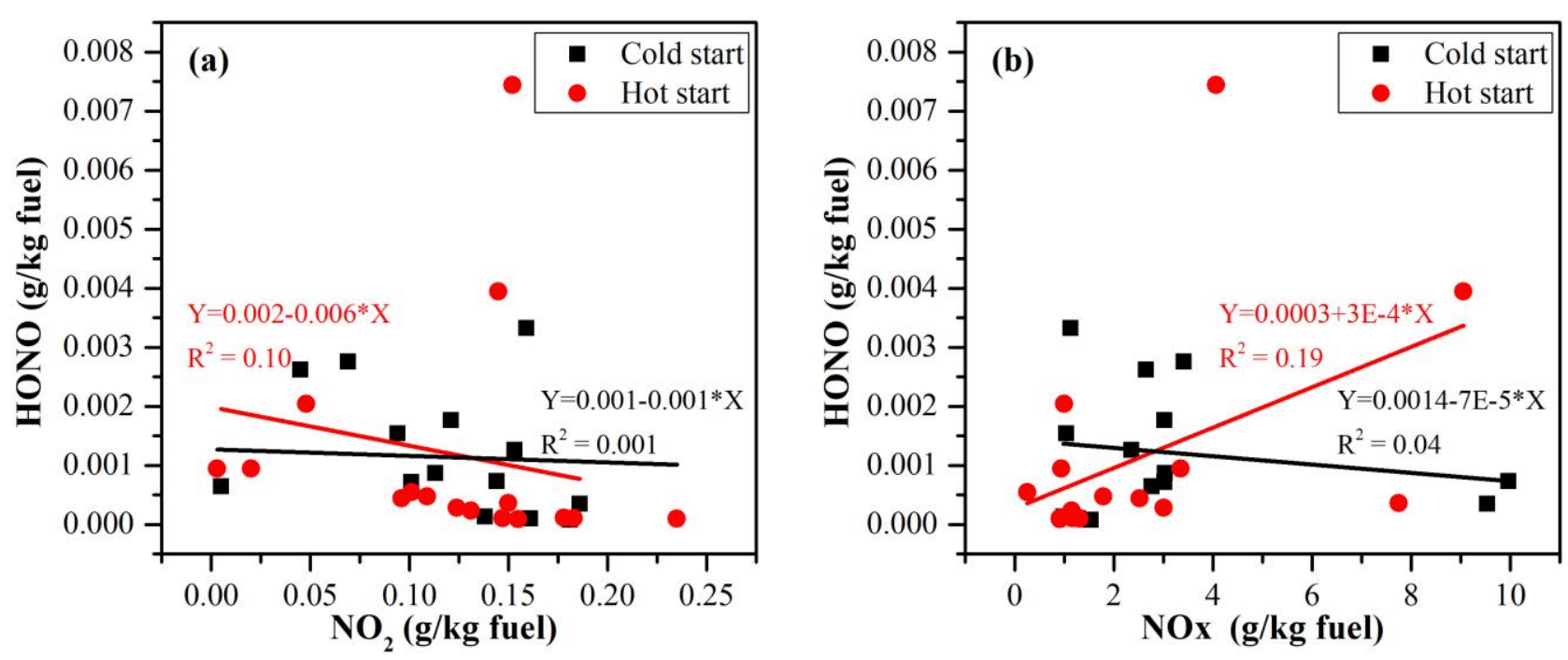

Figure S10. (a) Linear regressions for $\mathrm{HONO}$ EFs and $\mathrm{NO}_{2} \mathrm{EFs}$ and (b) Linear regressions for HONO EFs and NOx EFs of LDGVs tested at two start modes: hot start and cold start

\section{References}

(1) Pitts, J. N.; Biermann, H. W.; Winer, A. M.; Tuazon, E. C. Spectroscopic Identification and Measurement of Gaseous Nitrous Acid in Dilute Auto Exhaust. Atmos. Environ. 1984, 18 (4), 847854. https://doi.org/10.1016/0004-6981(84)90270-1.

(2) Kirchstetter, T. W.; Harley, R. A.; Littlejohn, D. Measurement of Nitrous Acid in Motor Vehicle Exhaust. Environ. Sci. Technol. 1996, 30 (9), 2843-2849. https://doi.org/10.1021/es960135y.

(3) Kurtenbach, R.; Becker, K. H.; Gomes, J. A. G.; Kleffmann, J.; Lörzer, J. C.; Spittler, M.; Wiesen, P.; Ackermann, R.; Geyer, A.; Platt, U. Investigations of Emissions and Heterogeneous Formation of HONO in a Road Traffic Tunnel. Atmos. Environ. 2001, 35 (20), 3385-3394. https://doi.org/10.1016/S1352-2310(01)00138-8.

(4) Kleffmann, J.; Gavriloaiei, T.; Hofzumahaus, A.; Holland, F.; Koppmann, R.; Rupp, L.; Schlosser, E.; Siese, M.; Wahner, A. Daytime Formation of Nitrous Acid: A Major Source of OH Radicals in a Forest. Geophys. Res. Lett. 2005, 32 (5), 1-4. https://doi.org/10.1029/2005GL022524. 
(5) Rappenglück, B.; Lubertino, G.; Alvarez, S.; Golovko, J.; Czader, B.; Ackermann, L. Radical Precursors and Related Species from Traffic as Observed and Modeled at an Urban Highway Junction. J. Air Waste Manag. Assoc. 2013, 63 (11), 1270-1286.

https://doi.org/10.1080/10962247.2013.822438.

(6) Xu, Z.; Wang, T.; Wu, J.; Xue, L.; Chan, J.; Zha, Q.; Zhou, S.; Louie, P. K. K.; Luk, C. W. Y. Nitrous Acid (HONO) in a Polluted Subtropical Atmosphere: Seasonal Variability, Direct Vehicle Emissions and Heterogeneous Production at Ground Surface. Atmos. Environ. 2015, 106 (x), 100-109. https://doi.org/10.1016/j.atmosenv.2015.01.061.

(7) Yang, Q.; Su, H.; Li, X.; Cheng, Y.; Lu, K.; Cheng, P.; Gu, J.; Guo, S.; Hu, M.; Zeng, L.; Zhu, T.; Zhang, Y. Daytime HONO Formation in the Suburban Area of the Megacity Beijing, China. Sci. China Chem. 2014, 57 (7), 1032-1042. https://doi.org/10.1007/s11426-013-5044-0.

(8) Liang, Y.; Zha, Q.; Wang, W.; Cui, L.; Lui, K. H.; Ho, K. F.; Wang, Z.; Lee, S. C.; Wang, T. Revisiting Nitrous Acid (HONO) Emission from on-Road Vehicles: A Tunnel Study with a Mixed Fleet. J. Air Waste Manag. Assoc. 2017, 67 (7), 797-805. https://doi.org/10.1080/10962247.2017.1293573.

(9) Kramer, L. J.; Crilley, L. R.; Adams, T. J.; Ball, S. M.; Pope, F. D.; Bloss, W. J. Nitrous Acid (HONO) Emissions under Real-World Driving Conditions from Vehicles in a UK Road Tunnel. Atmos. Chem. Phys. 2020, 20 (9), 5231-5248. https://doi.org/10.5194/acp-20-5231-2020.

(10) Nakashima, Y.; Kajii, Y. Determination of Nitrous Acid Emission Factors from a Gasoline Vehicle Using a Chassis Dynamometer Combined with Incoherent Broadband Cavity-Enhanced Absorption Spectroscopy. Sci. Total Environ. 2017, 575, 287-293. https://doi.org/10.1016/j.scitotenv.2016.10.050.

(11) Trinh, H. T.; Imanishi, K.; Morikawa, T.; Hagino, H.; Takenaka, N. Gaseous Nitrous Acid (HONO) 
and Nitrogen Oxides (NOx) Emission from Gasoline and Diesel Vehicles under Real-World Driving Test Cycles. J. Air Waste Manag. Assoc. 2017, 67 (4), 412-420.

https://doi.org/10.1080/10962247.2016.1240726.

(12) Liu, Y.; Lu, K.; Ma, Y.; Yang, X.; Zhang, W.; Wu, Y.; Peng, J.; Shuai, S.; Hu, M.; Zhang, Y. Direct Emission of Nitrous Acid (HONO) from Gasoline Cars in China Determined by Vehicle Chassis Dynamometer Experiments. Atmos. Environ. 2017, 169, 89-96. https://doi.org/10.1016/j.atmosenv.2017.07.019.

(13) Xue, J.; Tian W, K.; Zhang, Q. Research on Development of Vehicle Emission Inventory from motor vehicle in Hangzhou and Study on Its influnce on Air Quality. Reserch of Environmental Secience. 2010, 23(05), 613-618. 10.13198/j.res.2010.05.89.xuejp.014.

(14) Xian-jun, H. O. U.; Tian-tian, W.; Jun, Y. I. N.; Fu-ming, P.; Fu-wu, Y. A. N.; Xian-an, Z.; Engineering, A.; Aviation, P.; Co, E. Research on Status Quo of Emissions from Diesel Vehicles in Shenzhen. Auto Mob. Sci. Technol. 2010, 11-14.

(15) Guo, J.; Ge, Y.; Hao, L.; Tan, J.; Li, J.; Feng, X. On-Road Measurement of Regulated Pollutants from Diesel and CNG Buses with Urea Selective Catalytic Reduction Systems. Atmos. Environ. 2014, 99 (x), 1-9. https://doi.org/10.1016/j.atmosenv.2014.07.032.

(16) Yao, Z.; Wu, B.; Wu, Y.; Cao, X.; Jiang, X. Comparison of NOx Emissions from China III and China IV In-Use Diesel Trucks Based on on-Road Measurements. Atmos. Environ. 2015, 123, 1-8. https://doi.org/10.1016/j.atmosenv.2015.10.056. 